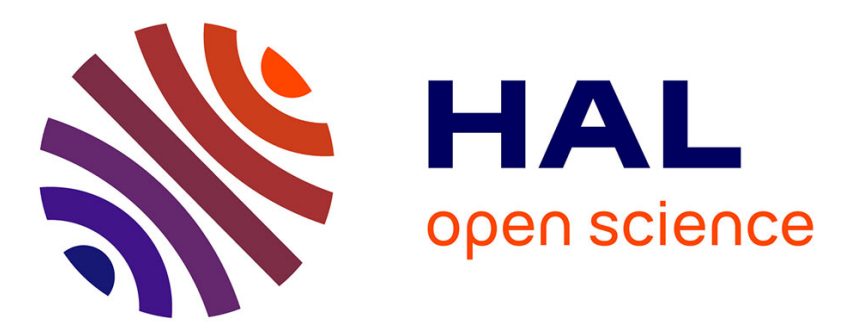

\title{
Design of haptic guides for pre-positioning assistance of a comanipulated needle
}

\author{
Hadrien Gurnel, Maud Marchal, Laurent Launay, Luc Beuzit, Alexandre \\ Krupa
}

\section{- To cite this version:}

Hadrien Gurnel, Maud Marchal, Laurent Launay, Luc Beuzit, Alexandre Krupa. Design of haptic guides for pre-positioning assistance of a comanipulated needle. SMC 2019 - IEEE International Conference on Systems, Man, and Cybernetics, Oct 2019, Bari, Italy. pp.478-485, 10.1109/SMC.2019.8914395 . hal-02387192

\section{HAL Id: hal-02387192 \\ https://hal.inria.fr/hal-02387192}

Submitted on 29 Nov 2019

HAL is a multi-disciplinary open access archive for the deposit and dissemination of scientific research documents, whether they are published or not. The documents may come from teaching and research institutions in France or abroad, or from public or private research centers.
L'archive ouverte pluridisciplinaire HAL, est destinée au dépôt et à la diffusion de documents scientifiques de niveau recherche, publiés ou non, émanant des établissements d'enseignement et de recherche français ou étrangers, des laboratoires publics ou privés. 


\title{
Design of haptic guides for pre-positioning assistance of a comanipulated needle
}

\author{
Hadrien Gurnel ${ }^{1,2,3,4,5}$, Maud Marchal ${ }^{1,2,3,4,5}$, Laurent Launay ${ }^{5}$, Luc Beuzit ${ }^{5,6}$, Alexandre Krupa $a^{1,3,4,5}$
}

\begin{abstract}
In minimally-invasive procedures like biopsy, the physician has to insert a needle into the tissues of a patient to reach a target. Currently, this task is mostly performed manually and under visual guidance. However, manual needle insertion can result in a large final positioning error of the tip that might lead to misdiagnosis and inadequate treatment. A way to solve this limitation is to use shared control; a gestureassistance paradigm that combines the cognitive skills of the operator with the precision, stamina and repeatability of a robotic or haptic device. In this paper, we propose to assist the physician with a haptic device that holds the needle and generates mechanical guides during the phase of manual needle pre-positioning. In the latter, the physician has to place the tip of the needle on a planned entry point, with a pre-defined angle of incidence. From this pre-operative information and also from intra-operative measurements, we propose to generate haptic cues, known as virtual fixtures, to guide the physician towards the desired position and orientation of the needle. It takes the form of five haptic guides, each one implementing virtual fixtures. We conducted a user study where those guides were compared to the unassisted reference gesture. The most constraining guide, in terms of assisted degrees of freedom, was highlighted as the one that provides the best results in terms of performance and user experience.
\end{abstract}

\section{INTRODUCTION}

Percutaneous needle insertion is frequently used for diagnosis (e.g. biopsy) or treatment (e.g. brachytherapy or localised tumour ablation). The outcome of those minimallyinvasive procedures (MIPs) depends almost exclusively on the accuracy of the placement of the needle tip on an anatomical target. Inaccurate positioning may require the physician to remove the needle from the tissues and perform the insertion again, thus increasing the duration of the intervention and resulting in greater discomfort and stress for the patient. Currently, needle insertion is mostly performed manually. Even though the physician is assisted with the visual feedback provided by imaging modalities such as Magnetic-Resonance Imaging (MRI), Computed Tomography (CT) or ultrasound, manual needle insertion is prone to error for several reasons. As presented by Abolhassani et al. [1], through the entry point, target visibility, target access and tool maneuverability are reduced. Added to poor technique or insufficient skills, this could severely impair the positioning accuracy. Then, the interaction forces between the needle and the tissues, which are modeled by Okamura et al. [2], make it difficult to correct the trajectory of the needle once it is inside soft tissues. Therefore, correct pre-positioning of the needle is essential for success.

Mail to: (maud.marchal, alexandre.krupa)@irisa.fr

1 Univ Rennes, ${ }^{2}$ INSA Rennes, ${ }^{3}$ IRISA, ${ }^{4}$ Inria, ${ }^{5}$ IRT $\mathrm{b}<>$ com, 6 CHU Rennes
To make needle pre-insertion more precise and reliable, one idea is to provide the physician with robotic assistance, a solution which was already successfully applied to prostatecancer diagnosis and treatment, as surveyed by Kaye et al. [3]. Within this context, haptics and shared control are promising. Indeed, haptic feedback can either help restore the sense of touch of the physician or provide guidance cues. Shared control combines the cognitive skills of the physician with the precision, stamina and repeatability of a robotic or haptic device. As a result, the user is kept in the loop and his/her dexterity is enhanced. In this study, we propose to assist the physician with shared robotic control, in order to perform the insertion in cooperation with a haptic device that holds the needle and generates mechanical guides. In the literature, shared control comes in two forms, i.e teleoperation and comanipulation. We investigate comanipulation because, compared to teleoperation, it enables closer proximity between the physician and the patient.

Guidance can be provided to the physician in the form of haptic cues yielding information about the current state of the instrument and/or its positioning error with respect to its planned trajectory. These cues are known as Virtual Fixtures (VFs), and they are computer-generated forces conveyed to the operator as feedback during a procedure. They were introduced by Rosenberg [4] for a peg-in-hole task in a teleoperation scenario. Hence, in this paper, we base our contribution on the use of VFs.

\section{RELATED WORK}

Shortly after their debut, VFs were introduced into the operating room. They fall in two categories, Guiding Virtual Fixtures (GVFs) and Forbidden-Region Virtual Fixtures (FRVFs), as surveyed by Bowyer et al. [5]. A GVF constrains the motion of a medical tool along a desired trajectory, pulling it back if the user moves it away. A FRVF defines forbidden areas, in which the tool should not penetrate. They prevent the instrument from adopting poses that could be hazardous to the safety of the intervention. Motion is free outside of those areas.

To the best of our knowledge, the first medical application of VFs was within the field of robot-assisted knee surgery and it is attributed to Ho et al. [6]. The authors define virtual walls to keep the milling tool within an authorised region. Later, this work was implemented in the ACROBOT device [7]. Since then, VFs have been an active topic of research. They were applied to teleoperated systems, for instance. Xiong et al. [8] present a robotic mechanism dedicated to path-following and tumour-targeting tasks with a needle, in 
interventional radiology. They compute both a GVF and a FRVF in the form of a potential field to guide the user towards a path or a target and keep him/her away from simulated obstacles. A haptic assistance combining a GVF and a FRVF was also designed by Meli et al. [9] for teleoperated needle-insertion procedures. The GVF provides torque feedback to the user to correct the pose of the needle, while the FRVF produces a vibration when the desired insertion depth is reached. Also, contrary to the previous two contributions, the authors conducted a user-experience study in addition to a performance study, through tissuediscrimination and targeting tasks.

Aside from teleoperated devices, various comanipulated contributions were proposed to assist needle or laparoscopicinstrument manipulation with VFs. Vitrani et al. [10] use a commercial haptic device to convey the forces applied at the tip of a laparoscopic instrument to the user through GVFs and FRVFs. The latter are modeled as linear springs. This work was extended to instrument positioning by Chalard et al. [11], where the deformation of the entry point is taken into account to refine the Jacobian matrix between the proximal and the distal ends of the instrument. Gijbels et al. [12] introduce a robotic manipulator dedicated to assisting the insertion of a needle during retinal-vein cannulation. Their system includes a mechanical Remote Centre of Motion (RCM), which limits the lateral translations of the needle. Some haptic feedback scales the velocity of the needle, but also filters hand tremor. However, those haptic cues do not provide indications about the location of the target. Wartenberg et al. [13] present a robotic assistance device for prostate interventions involving the insertion of a beveled-tip needle. With this approach, needle pre-positioning and preorienting on the entry point are automatically controlled by the system. Inside soft tissues, the axial rotation of the needle is controlled by the system, thanks to two forces sensors. The insertion velocity of the tip is collaboratively controlled by the physician and the robotic device. Even though this approach takes needle bending into account, the user can only influence one degree of freedom (DoF) of the needle.

In this paper, we propose to assist the physician during comanipulated needle pre-positioning and pre-orienting on a target, with a desired angle of incidence. Those are key steps for accurately positioning the tip. Indeed, once the needle is inserted in the tissues of the patient, it is difficult to change its orientation without pulling it back. Several research groups investigated needle pre-positioning assistance, mainly with visual guidance. For instance, $\mathrm{Xu}$ et al. [14] developed a smartphone augmented-reality application to provide visual guidance to the surgeon during the phase of angle selection of manual pre-insertion. With the Imactis ${ }^{\mathrm{TM}}$ commercial CTnavigation system, Durand et al. [15] enhanced CT-guided interventions with a visual feedback of the trajectory of the needle before the insertion, thanks to electro-magnetic tracking. Another visual-guidance device for needle preinsertion assistance was proposed by Grasso et al. [16]. It combines infrared tracking and image processing. Though, with those devices, the assistance of manual needle pre- insertion is only image-based, which requires the user to keep an eye on a screen at all times. Some automatic preinsertion devices also exist, such as the ones presented by Hungr et al. [17], Wartenberg et al. [13] or the commercial robots NeuroMate ${ }^{\mathrm{TM}}$ (Renishaw) [18] and Maxio ${ }^{\mathrm{TM}}$ (Perfint Healthcare, India) [19]. However, they do not keep the physician directly in the control loop during this phase of needle insertion and this can result in important acceptability issues for the clinician [20]. A comanipulated solution was proposed by Vitrani et al. [21] to position a transrectal ultrasound probe. The authors present a clinical validation of the free and locked modes of the Apollo cobotic device. The locked mode enables the physician to safely release the probe while he/she manually inserts a needle along a physical guide attached to the probe. Thus, Apollo acts as a needle pre-positioning assistant. Though, no haptic guidance is used to position the probe on the entry point.

Contribution: in this paper, we present the design and comparison of 5 haptic guides (FTip, TTip, FTTip, FTATip, TEff) dedicated to comanipulated-needle pre-insertion. With respect to the image-based assistance methods presented earlier, our haptic guides represent an additional layer of sensory information, conveyed to the user through kinaesthetic feedback. They are designed to enable the physician to correct his/her gesture in a more accurate, intuitive and comfortable way, without constantly having to look at a screen. Those approaches produce haptic cues on up to 5 DoFs of the needle. They implement GVFs, to guide the user towards the entry point and the correct orientation. In a context of needle pre-insertion, FRVFs are less relevant, because they would prevent the physician from colliding with obstacles, which is unlikely to happen before the needle is inserted in soft tissues. We also present a user study to evaluate the performances of the five guides and the unassisted reference gesture (Ref) in a pre-positioning task. The remaining of this paper is organised as follows. Details about each approach are provided within Sec.III. Sec. IV introduces our user study, whose results are exhibited and discussed in Sec. V and VI, respectively.

\section{Methods}

\section{A. General information}

We designed five haptic guides, denoted by FTip, TTip, FTTip, FTATip, TEff and illustrated in Fig. 1. Each of them produces a $6 \times 1$ force-feedback vector $f_{\text {eff }}$ ( 3 forces and 3 torques) that is applied to the end-effector frame of the haptic device, which holds the needle. This force vector is computed from the relative pose error between the current measure of the pose of the needle-tip frame (or the current pose of the end-effector frame of the haptic device) and the desired pose of the entry-point frame. As shown in Fig. 1, the desired entry-point frame is centered on the entry point and its $\mathrm{z}$ axis corresponds to the desired angle of incidence to reach. The force-feedback vector is conveyed to the user in real-time by the haptic device, to guide him/her towards the target with the desired angle of incidence. 


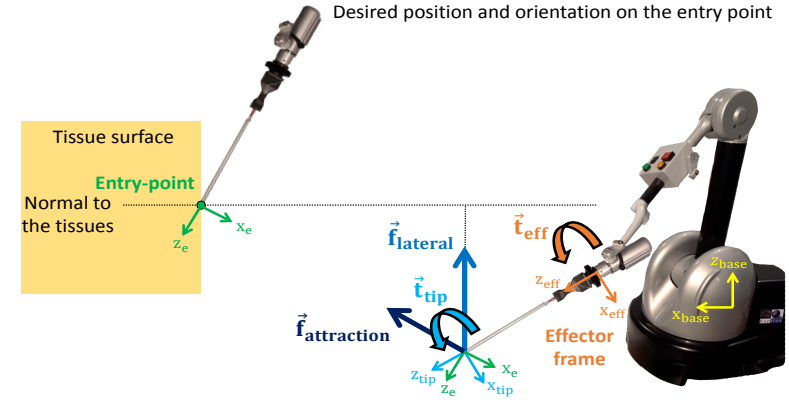

Fig. 1. The proposed five haptic guides. The blue arrows represent the needle-tip frame, as well as forces and a torque expressed in this frame. FTip, TTip, FTTip and FTATip implement one or a combination of those forces and this torque. The orange arrows represent the end-effector frame of the haptic device and a torque expressed in this frame, which is implemented by TEff.

All the guides rely on the use of one or more centered sigmoid functions that control the level of stiffness of the haptic assistance along every component of the force-feedback vector. The generic expression of the centered sigmoid is presented in equation (1). In our approach, we consider two scalar sigmoid functions that provide generic expressions for any force component $f$ and torque component $\tau$ of the forcefeedback vector:

$$
f=F_{\max } \frac{1-e^{-\beta_{f} d}}{1+e^{-\beta_{f} d}} \quad \tau=T_{\max } \frac{1-e^{-\beta_{t} \theta}}{1+e^{-\beta_{t} \theta}}
$$

In case of a translational DoF, $d$ corresponds to the error with respect to the desired translation computed by the guide and, similarly, $\theta$ is the angular error for a rotational DoF. For safety purposes, $F_{\max }$ and $T_{\max }$ define the maximum values of the force and torque components the haptic guide can deliver to the user. The shape parameter $\beta$ (with $\beta=\beta_{f}$ for forces and $\beta=\beta_{t}$ for torques) of the sigmoid is set to control the level of stiffness of the haptic assistance. Low shape-parameter values lead to smooth and continuous haptic feedback, so the user constantly feels some feedback, even for low deviations with respect to the guide reference. High values of $\beta$ result in rapidly-increasing forces. As a result, the user feels almost no feedback for low deviations, but quickly reaches the maximum value of the force or torque intensity as the targeting error increases. Hence, a trade-off between comfort and accuracy arises. Indeed, with smooth feedback, manipulating the needle is comfortable, but the user does not feel much force from the haptic device until the deviation is significant, which might lead to targeting inaccuracies. On the contrary, with stiff feedback, he/she is significantly constrained, and potentially more accurate, but stiff feedback may cause fatigue and muscle pain, which is undesirable. Balance between comfort and accuracy is, thus, found by empirically setting the value of $\beta$ for each haptic guide.

In practice, the feedback force $f_{\text {eff }}$ can only be rendered to the user through the handle of the haptic device and thus, it has to be mapped to the end-effector frame. However, depending on the guides we will present in the next section, the force vector will be computed either in the needletip frame as $\mathbf{f}_{\text {tip }}$ or directly in the end-effector frame as $f_{\text {eff }}$. Each non-zero component of $f_{\text {tip }}$ or $\mathbf{f}_{\text {eff }}$ corresponds to a constrained DoF of the needle. If the force-feedback vector is computed in the needle-tip frame, the following transformation is required to map it to the end-effector frame:

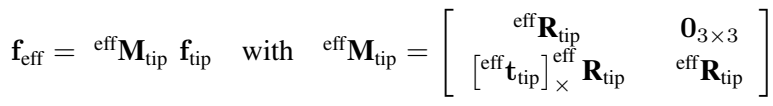

where ${ }^{\text {eff }} \mathbf{t}_{\text {tip }}$ and ${ }^{\text {eff }} \mathbf{R}_{\text {tip }}$ are the $3 \times 1$ vector and $3 \times 3$ rotation matrix representing the position and orientation of the needle-tip frame with respect to the end-effector frame. $\left.{ }^{\mathrm{eff}} \mathbf{t}_{\mathrm{tip}}\right]_{\times}$is the skew symmetric matrix built from the translation vector.

In the remaining of this paper, the yaw, pitch and roll angles correspond to rotations around the $\mathrm{x}, \mathrm{y}$ and $\mathrm{z}$ axes of the needle-tip frame, respectively.

\section{B. Lateral force applied to the tip of the needle (FTip)}

As illustrated by Fig. 1, this haptic guide constrains the position of the tip of the needle, to keep it close to the normal of the tissue surface that crosses the entry point. As a result, the tip can be translated along the normal, but as soon as it deviates from it, a force is generated to pull it back. Translations along the $\mathrm{z}$ axis and rotations around the yaw, pitch and roll axes of the tip frame are free. Therefore, this approach constrains two DoFs of the needle and this is achieved by applying a lateral force $\mathbf{f}_{\text {tip }}=\left(f_{t_{x}}, f_{t_{y}}, 0,0,0,0\right)^{T}$ to the tip. The expressions of $f_{t_{x}}$ and $f_{t_{y}}$ are similar and given by equation (1). In this case, deviation $d$ represents either the $\mathrm{x}$ or $\mathrm{y}$ component of the orthogonal projection of the tip on the normal of the tissue surface. Velocity damping is applied to the force vector to insure stability. To this end, a coefficient $\zeta_{f}$ specific to forces is added to the two constrained DoFs and the resulting forcefeedback vector expressed in the needle-tip frame becomes:

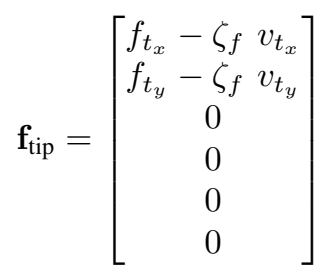

where $v_{t_{x}}$ and $v_{t_{y}}$ correspond to the $\mathrm{x}$ and $\mathrm{y}$ translational components of the $6 \mathrm{x} 1$ velocity vector of the needle-tip frame $\mathbf{v}_{\text {tip }}=\left(v_{t_{x}}, v_{t_{y}}, v_{t_{z}}, \omega_{t_{y a w}}, \omega_{t_{\text {pitch }}}, \omega_{t_{\text {roll }}}\right)^{T}$ expressed in the needle-tip frame. The force-feedback vector is then mapped to the end-effector frame according to equation (2).

\section{Torque applied to the tip of the needle (TTip)}

The objective of this guide is to constrain the orientation of the needle to the desired angle of incidence, regardless of the current position of the tip. For this reason, TTip applies a torque to the needle, around the yaw and 
pitch axes of the needle-tip frame. It is denoted by $\mathbf{f}_{\text {tip }}=$ $\left(0,0,0, t_{t_{y a w}}, t_{t_{\text {pitch }}}, 0\right)^{T}$, as presented in Fig. 1. All the translations and the rotation around the roll axis of the needle are free. The expressions of the torque components of the output force-feedback vector, $t_{t_{y a w}}$ and $t_{t_{p i t c h}}$, are given by equation (1). In this case, deviation $\theta$ corresponds to the yaw and pitch angular deviations of the entry-point frame relative to the needle-tip frame. Similarly to FTip, velocity-damping is added to the non-zero components of the force-feedback vector, but with a damping coefficient specific to torques and denoted by $\zeta_{t}$. Consequently, the expression of the output force-feedback vector is provided by equation (4). It is then mapped to the end-effector frame, according to equation (2).

$$
\mathbf{f}_{\text {tip }}=\left[\begin{array}{c}
0 \\
0 \\
0 \\
t_{t_{y a w}}-\zeta_{t} \omega_{t_{y a w}} \\
t_{t_{\text {pitch }}}-\zeta_{t} \omega_{t_{\text {pitch }}} \\
0
\end{array}\right]
$$

D. Lateral force and torque applied to the tip of the needle (FTTip)

This approach combines FTip and TTip, in order to constrain both the position and the orientation of the needle. This is shown in Fig. 1. Only translations along the $\mathrm{z}$ axis and rotations along the roll axis of the needle-tip frame are free. For each non-zero component of the force-feedback vector, deviations $d$ and $\theta$ are computed with a method similar to FTip and TTip. Velocity-damping is also added. The expression of the force-feedback vector is given by equation (5). It is then mapped to the end-effector frame, according to equation (2).

$$
\mathbf{f}_{\text {tip }}=\left[\begin{array}{c}
f_{t_{x}}-\zeta_{f} v_{t_{x}} \\
f_{t_{y}}-\zeta_{f} v_{t_{y}} \\
0 \\
t_{t_{y a w}}-\zeta_{t} \omega_{t_{y a w}} \\
t_{t_{\text {pitch }}}-\zeta_{t} \omega_{t_{\text {pitch }}} \\
0
\end{array}\right]
$$

E. Lateral force, torque and attractive force applied to the tip of the needle (FTATip)

This haptic guide adds a smooth attractive force $\mathbf{f}_{\text {att }}=$ $\left(f_{a_{x}}, f_{a_{y}}, f_{a_{z}}, 0,0,0\right)^{T}$ to the force-feedback vector of FTTip. It is computed in the needle-tip frame and oriented towards the entry point, as illustrated by Fig. 1. With FTATip, only the rotations around the roll axis of the needle-tip frame are free. The behaviour of the attractive force is described by equation (6), where $\mathbf{p}$ is a $3 \times 1$ vector representing the position of the entry point expressed in the needle-tip frame and $r>0$ is the radius of a sphere centered on the entry point. Outside the sphere, the force attracts the needle towards the target with a constant intensity, equal to a ratio $\alpha \in[0,1]$ of the maximum force intensity, $F_{\max }$. Inside the sphere, the magnitude of the attractive force smoothly decreases, so as to reduce the momentum of the needle when it is close to the entry point. As for $f_{t_{x}}, f_{t_{y}}, t_{t_{y a w}}$ and $t_{t_{\text {pitch }}}$, they are computed with the same method as for FTip and TTip. Finally, the expression of the force-feedback vector is given by equation (7). Velocity-damping is also added. It is then mapped to the end-effector frame, according to equation (2).

$$
\begin{aligned}
& \mathbf{f}_{\mathrm{att}}= \begin{cases}\alpha F_{\max } \sqrt{\frac{\|\mathbf{p}\|}{r}} \frac{\mathbf{p}}{\|\mathbf{p}\|} & , \text { if }\|\mathbf{p}\| \leq r \\
\alpha F_{\max } \frac{\mathbf{p}}{\|\mathbf{p}\|} & , \text { otherwise }\end{cases} \\
& \mathbf{f}_{\text {tip }}=\left[\begin{array}{c}
f_{t_{x}}+f_{a_{x}}-\zeta_{f} v_{t_{x}} \\
f_{t_{y}}+f_{a_{y}}-\zeta_{f} v_{t_{y}} \\
f_{a_{z}}-\zeta_{f} v_{t_{z}} \\
t_{t_{y a w}}-\zeta_{t} \omega_{t_{y a w}} \\
t_{t_{\text {pitch }}}-\zeta_{t} \omega_{t_{\text {pitch }}} \\
0
\end{array}\right]
\end{aligned}
$$

\section{F. Torque applied to the effector of the haptic device (TEff)}

As shown in Fig. 1, this method ensures the needle always points towards the entry point. The axis of the needle can be seen as the radius of a sphere, whose centre is the entry point. This behaviour is obtained by applying a torque to the yaw and pitch axes of the end-effector frame of the haptic device. The force vector is denoted by $\mathbf{f}_{\text {eff }}=$ $\left(0,0,0, t_{e_{y a w}}, t_{e_{\text {pitch }}}, 0\right)^{T}$. In this case, the deviation $\theta$ of the sigmoid, presented in equation (1), corresponds either to the yaw or to the pitch angle between the axis of the needle and the 3D straight line that connects the origin of the end-effector frame and the entry point. The expression of the force-feedback vector is given by equation (8), where $\omega_{e_{y a w}}$ and $\omega_{e_{\text {pitch }}}$ correspond to the yaw and pitch rotational components of the $6 \times 1$ velocity vector of the end-effector frame $\mathbf{v}_{\text {eff }}=\left(v_{e_{x}}, v_{e_{y}}, v_{e_{z}}, \omega_{e_{y a w}}, \omega_{e_{p i t c h}}, \omega_{e_{\text {roll }}}\right)^{T}$ expressed in the end-effector frame.

$$
\begin{gathered}
\mathbf{f}_{\mathrm{eff}}=\left[\begin{array}{c}
0 \\
0 \\
0 \\
t_{e_{y a w}}-\zeta_{t} \omega_{e_{y a w}} \\
t_{e_{p i t c h}}-\zeta_{t} \omega_{e_{p i t c h}} \\
0
\end{array}\right] \\
\text { IV. USER STUDY }
\end{gathered}
$$

We conducted a user study to compare the performance of the five haptic guides, as well as the unassisted reference gesture. The evaluation was focused on the measurement of the participant performance for pre-positioning a needle before its insertion into soft tissues. The task consisted in positioning a needle under a desired orientation within a given accuracy. We measured the time needed by the participants to complete the task. Our main hypotheses are the following ones:

- $\mathbf{H}_{\mathrm{A}}$ : the more the user is constrained by the haptic guide, the faster he/she will complete the positioning task.

- $\mathbf{H}_{\mathbf{B}}$ : the assistance applied to the tip of the needle is more relevant than the assistance applied to the end-effector for pre-positioning the needle before its insertion. 

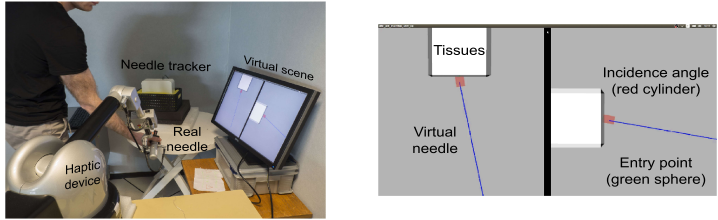

Fig. 2. Description of the experimental setup: (a) the participant is manipulating the needle, which is attached to a 6-DoF haptic device, (b) the virtual scene is made of an entry point (represented by a small green sphere on the tissue surface) and a desired trajectory (represented by a red cylinder). The participant had to position the tip of the needle on the position of the entry point, with the desired orientation.

a) Population: twelve unpaid participants, recruited among students and staff, volunteered for the user study (3 females, 9 males; age: mean $=27.3$, standard deviation $(\mathrm{SD})=3.4, \min =22, \max =33$ ). They were all naive to the purpose of the experiment, right-handed, had normal or correct-to-normal vision, and gave written and informed consent. None were experts in needle manipulation nor in haptics.

b) Experimental setup: the participants handled a 150 $\mathrm{mm} 18 \mathrm{G}$ instrumented needle that was tracked electromagnetically by an Aurora ${ }^{\mathrm{TM}}$ device, (Northern Digital Inc., Ontario, Canada). The needle was attached to a 6-DoF haptic device (Virtuose ${ }^{\mathrm{TM}} 6 \mathrm{D}$, Haption [22], France) (see Fig. 2). The electro-magnetic tracker measured the 5-DoF pose of the instrumented needle at a frequency of $66 \mathrm{~Hz}$. Contrary to imaging modalities such as ultrasound, the Aurora ${ }^{\mathrm{TM}}$ provides a direct measure of the pose of the needle. In this setup, the needle was mounted on the end-effector of the haptic device, via a 3D-printed needle holder, which was grabbed by the right hand of the participants for needle manipulation. Simultaneously, a computer screen displayed top and side views of a virtual scene composed of the virtual needle (represented as a blue line), the surface of the tissues (white cube), the target (green sphere) and the desired angle of incidence (red cylinder). The update of the needle position in the virtual scene relied on the measurements collected from the haptic device and the needle tracker. The position of the virtual needle was synchronised in real-time with the position of the real needle. During the user study, the position of the entry point was pre-defined by the operator, who recorded it before the experiment, thanks to the instrumented needle.

c) Electro-magnetic-field interferences: to assess the impact of the metallic structure of the haptic device on tracking accuracy, we acquired two paired point-sets. Each pair of points was obtained by probing twice the same position and orientation in space with the Aurora ${ }^{\mathrm{TM}}$ needle, either connected to the haptic device (test condition) or unconnected (reference condition). The pairs of points each represented a different position and orientation of the needle. 30 different pairs were probed. We then compared the two homologous point sets. This showed an average distance of $0.78 \mathrm{~mm}$ between corresponding points, a position error of $0.46 \mathrm{~mm}(\mathrm{SD}=0.47 \mathrm{~mm})$ along the $\mathrm{x}$ axis of the frame of the tracker, $0.53 \mathrm{~mm}(\mathrm{SD}=0.50 \mathrm{~mm})$ along the $y$ axis and $0.54 \mathrm{~mm}(\mathrm{SD}=0.46 \mathrm{~mm})$ along the $\mathrm{z}$ axis. Typical targeting accuracies for interventions such as biopsy are in the range of 2 to $4 \mathrm{~mm}$. As we do not consider micro-surgery applications, $0.78 \mathrm{~mm}$ is acceptable and the influence of the haptic device on the electro-magnetic field is negligible.

d) Calibration: to update the virtual scene with the real needle position, a calibration was performed before the experiment. The coordinates of the components of our experimental setup are expressed in the base frame of the haptic device, which acts as the world frame of the setup. The positions of the needle tip and the entry point are provided by the electro-magnetic tracker and need to be mapped to the base frame of the haptic device. The calibration process is divided into two stages. First, the 3D position of the tip of the needle relative to the end-effector frame of the haptic device is estimated. To this end, pivot calibration, and more specifically, an adaptation of the algebraic two-step variant, presented in [23], is implemented. The 3D coordinates of the tip of the needle are obtained by probing a unique pivot point in space with the needle, with various poses of the endeffector. Every configuration of the end-effector yields an expression, similar to equation (9). ${ }^{b} \mathbf{x}_{t}$ and ${ }^{e} \mathbf{x}_{t}$ correspond to the constant $3 \mathrm{D}$ position vectors of the tip of the needle with respect to the base frame and the end-effector frame (the latter is the output of the first calibration), respectively. Those position vectors are constant because for every configuration of the effector, the tip is positioned on the same pivot point. ${ }^{b} \mathbf{R}_{e, i}$ and ${ }^{b} \mathbf{t}_{e, i}$ are the i-th rotation matrix and translation vector of the end-effector frame (i.e. in its i-th configuration) relative to the base frame. For $n$ configurations of the effector, $\mathrm{n}$ expressions similar to equation (9) are obtained. From this, ${ }^{e} \mathbf{x}_{t}$ corresponds to the vector that minimizes the sum of the squared differences of any pair $(i, j)$ of equations (9), as presented in Equation (10). The second stage of the calibration deals with the computation of the constant pose of the electro-magnetic tracker, relative to the base of the haptic device. This is done by registering two corresponding point clouds (paired-point registration), using VTK's Landmark Transform [24], which implements [25]. The first point cloud is expressed in the frame of the tracker and the second one in the base frame of the haptic device. They are obtained by sweeping the work-space with the needle. Once calibration is over, any point can be expressed in the base frame of the haptic device.

$$
\begin{gathered}
{ }^{b} \mathbf{x}_{t}={ }^{b} \mathbf{R}_{e, i}{ }^{e} \mathbf{x}_{t}+{ }^{b} \mathbf{t}_{e, i} \\
{ }^{e} \mathbf{x}_{t}=\min _{{ }^{e} \mathbf{x}_{t}} \sum_{i=0}^{n-1} \sum_{j=0}^{n-1}\left|\left({ }^{b} \mathbf{R}_{e, i}{ }^{e} \mathbf{x}_{t}+{ }^{b} \mathbf{t}_{e, i}\right)-\left({ }^{b} \mathbf{R}_{e, j}{ }^{e} \mathbf{x}_{t}+{ }^{b} \mathbf{t}_{e, j}\right)\right|^{2}
\end{gathered}
$$

e) Task description: the task corresponded to a prepositioning task of the needle at the surface of soft tissues before its insertion. The participants performed the experiment in a standing position. They were asked to place the tip of the needle on the green sphere of the virtual scene representing the entry point, and to give the axis of the needle a desired angle of incidence, represented by a red cylinder. 
The latter was achieved by keeping the blue line (needle) parallel to the red cylinder, in both views of the virtual scene. The red cylinder became green when the task was successful. To simulate the interaction with the tissues, a repulsive force, based on a mass-spring-damper model, was generated when the participants penetrated the white cube. During the targeting tasks, they had to reach acceptable position and orientation accuracies, as fast as possible. The thresholds for validating the accuracy of a given participant were set, thanks to preliminary tests with two physicians, both experts on needle manipulation (see next paragraph). Before each trial, the needle was placed at a reference position and the participant had to press a key to start the trial. Once the task was fulfilled, the participant could release the needle manipulator and take a break before the next trial. The whole experiment lasted around $1 \mathrm{~h} 45 \mathrm{~min}$.

f) Preliminary tests with physicians: the thresholds for assessing the accuracy of the participants were defined through preliminary tests involving two physicians, an interventional radiologist and an anaesthetist, both experts on needle manipulation. In those tests, the physicians performed the experiment without a time constraint. They had no accuracy thresholds and had to validate the trial themselves, when they deemed their accuracy correct. After discussion with the two physicians, it was decided that for the user study with twelve novice participants, the acceptable accuracies would be set to $1.5 \mathrm{~mm}$ for the position difference between the tip and the entry point, and $4^{\circ}$ for the angular deviation of the needle with respect to the desired angle of incidence. During the tests, the final accuracy of the participants was measured when they reached the acceptable position and orientation accuracy thresholds simultaneously.

g) Experimental design: three conditions are considered in our experimental design:

- $\mathbf{C}_{\mathbf{A}}$ is the haptic guide. It corresponds to one of the five guides presented in Sec. III or to the unassisted reference gesture, which consists in not using haptic assistance during needle manipulation.

- $\mathbf{C}_{\mathbf{B}}$ is the desired orientation of the needle. Five different angles of incidence were proposed: a straight one and four others, defined with respect to tissue frame (x and $\mathrm{y}$ are on the tissue surface, with $\mathrm{x}$ pointing down, and $\mathrm{z}$ is normal to the tissue surface and oriented towards the inside of the tissues). The angles of incidence corresponded to the different combinations of the yawangle values $\left(-7^{\circ}\right.$ or $\left.7^{\circ}\right)$ and pitch-angle values $\left(-10^{\circ}\right.$ or $\left.10^{\circ}\right)$.

- $\mathbf{C}_{\mathbf{C}}$ is the stiffness for haptic rendering: 3 different stiffnesses were proposed (low, average, high). There were obtained by manually setting the parameters defined in Sec. III, i.e. the shape parameter of the sigmoids $\left(\beta_{f}\right.$ for forces and $\beta_{t}$ for torques) and the force-and-torque velocity-damping coefficients, $\zeta_{f}$ and $\zeta_{t}$. The values for all these parameters are provided in Table I.

The participants performed all the trials for one guide (or the reference) before switching to another one. The order of the guides was counterbalanced between the participants,

\begin{tabular}{|l|l|l|l|l|l|l|}
\hline Parameter & FTip & TTip & FTTip & FTATip & TEff & Ref \\
\hline$\beta_{f}\left(m^{-1}\right)$ & $15 ; 30 ; 35$ & $\times$ & $15 ; 30 ; 35$ & $15 ; 30 ; 35$ & $\times$ & $0 ; 0 ; 0$ \\
$\zeta_{f}\left(s^{-1}\right)$ & $0.85 ; 2.2 ; 4$ & $\times$ & $0.85 ; 2.2 ; 4$ & $0.85 ; 2.2 ; 4$ & $\times$ & $0 ; 0 ; 0$ \\
$\beta_{t}\left(\mathrm{rad}^{-1}\right)$ & $\times$ & $1 ; 2 ; 2.5$ & $1 ; 1 ; 1.5$ & $1 ; 1 ; 1.5$ & $1 ; 2 ; 2.5$ & $0 ; 0 ; 0$ \\
$\zeta_{t}\left(m^{2} s^{-2}\right)$ & $\times$ & $0.01 ; 0.08 ; 0.2$ & $0.04 ; 0.04 ; 0.03$ & $0.04 ; 0.04 ; 0.03$ & $0.01 ; 0.08 ; 0.2$ & $0 ; 0 ; 0$ \\
\hline
\end{tabular}

TABLE I

SHAPE PARAMETERS AND VELOCITY-DAMPING COEFFICIENTS FOR FORCES AND TORQUES, FOR 3 STIFFNESSES (LOW, AVERAGE, HIGH)

using a circular permutation. For each guide, the participants had to perform 5 trajectories $\times 3$ stiffnesses (random order), twice for each, leading to a total of 30 trials per guide. Therefore, the total number of trials was 180 for the whole experiment.

h) Collected data: we measured the time needed by each participant to position the needle within the acceptable accuracy determined thanks to the preliminary user study. The participants also filled a subjective questionnaire after performing the task with each guide, answering the following questions using a 7-point Likert scale: (1) "What level of assistance did the guidance method provide you with?", (2) "How accurate was the guidance method?", (3) "How easy was it to use the guidance method?", (4) "How comfortable was the guidance method?", (5) "Did the guidance method help you accomplish the task quickly?", (6) "To what extent did haptic feedback help you complete the task?" and (7) "To what extent did visual feedback help you complete the task?". At the end of the experiment, the participants were also asked to choose the guidance method they preferred, the one they enjoyed the least, and to explain their decision.

\section{RESULTS}

\section{A. Pre-positioning task}

To study the time needed by the participants to perform the pre-positioning task, we used a mixed linear model on the collected data, with respect to the three independent variables $\mathbf{C}_{\mathbf{A}}, \mathbf{C}_{\mathbf{B}}$ and $\mathbf{C}_{\mathbf{C}}$ defined in the experimental design. The participants are considered as a random effect in the model. To control the over-dispersion of the residuals, the measured time was log-transformed in our analysis.

Our analysis of variance showed a significant effect of $\mathbf{C}_{\mathbf{A}}(F(5,2137)=58.44, p<0.001)$ and $\mathbf{C}_{\mathbf{B}}$ $(F(4,2137)=33.90, p<0.001)$. A post-hoc analysis on $\mathbf{C}_{\mathbf{A}}$ using a Tukey test revealed significant differences between the guide FTATip $(M=8.90 s)$ and all the other five guides: Ref $(M=14.15 s ; p<0.001)$, FTip $(M=13.99 s$, $p<0.001)$, TTip $(M=12.73 s, p<0.001)$, FTTip $(M=13.00 s, p<0.001)$, and TEff $(M=14.36 s$, $p<0.001)$. We found also a significant difference between Ref and TTip ( $p=0.02)$, TTip and TEff ( $p=0.004)$, and FTTip and TEff ( $p=0.03$ ). Fig. 3 summarizes the results for the different guides. Concerning the trajectories $\left(\mathbf{C}_{\mathbf{A}}\right)$, the post-hoc analysis revealed a significant difference between the horizontal trajectory and the other ones $(p<0.001$ for each of them, except the (Yaw $=-7^{\circ}$, Pitch $=10^{\circ}$ ) $(p=0.005)$. We found also a significant difference between this last trajectory $\left(\mathrm{Yaw}=-7^{\circ}, \mathrm{Pitch}=10^{\circ}\right)$ and the other ones 
$\left(p<0.001\right.$ for trajectories (Yaw $=-7^{\circ}$, Pitch $\left.=-10^{\circ}\right)$ and $\left(\right.$ Yaw $=7^{\circ}$, Pitch $\left.=-10^{\circ}\right), p=0.01$ for the trajectory $\left(\right.$ Yaw $=7^{\circ}$, Pitch $\left.=-10^{\circ}\right)$ ).

On average, the participants reached position accuracies of $1.34 \mathrm{~mm}(\mathrm{SD}=0.17 \mathrm{~mm})$ and orientation accuracies of $2.22^{\circ}\left(\mathrm{SD}=0.99^{\circ}\right)$. Thus, they were more accurate than the acceptable-accuracy thresholds by $10.67 \%$ for position and by $44.25 \%$ for orientation.

\section{B. Subjective questionnaire}

An analysis of variance on the subjective questionnaire with respect to the different guides showed a significant effect for the following criteria: Level of assistance $(F(5,55)=21.90, p<0.001)$, Accuracy $(F(5,55)=19.64, p<0.001)$, Ease of use $(F(5,55)=8.96, p<0.001)$, Comfort $(F(5,55)=4.78$, $p=0.001)$, Accomplishment help $(F(5,55)=26.63$, $p<0.001)$, Help of haptic feedback $(F(5,55)=25.21$, $p<0.001)$. We did not obtain any significant effect for the Help of visual feedback criterion $(F(4,2137)=1.06$, $p=0.39$ ). The box plots representing the answers of the different criteria are shown in Fig. 3.

The preferences of the users, collected at the end of the study, are summarised in Table II.

\begin{tabular}{|l|l|l|l|l|l|l|}
\hline Preferred guides & FTATip & TTip & FTTip & FTip & TEff & Ref \\
\hline Votes & $6(50 \%)$ & $4(33.3 \%)$ & $1(8.33 \%)$ & $1(8.33 \%)$ & $0(0 \%)$ & $0(0 \%)$ \\
\hline Least-preferred guides & Ref & TEff & FTip & FTTip & TTip & FTATip \\
\hline Votes & $6(50 \%)$ & $2(16.67 \%)$ & $1(8.33 \%)$ & $1(8.33 \%)$ & $1(8.33 \%)$ & $1(8.33 \%)$ \\
\hline
\end{tabular}

TABLE II

PREFERENCES OF THE 12 PARTICIPANTS BETWEEN THE GUIDES

\section{DISCUSSION}

The goal of the user study was to compare the five haptic guides and the unassisted reference gesture from performance and user-experience perspectives. Ultimately, the optimal haptic assistance should be accurate, fast and comfortable.

On the performance side, the accuracy was imposed during the tasks and the objective results showed significant improvement of the execution time when haptic guidance was active, compared to the unassisted tasks. This validates $\mathbf{H}_{\mathbf{A}}$. This result is especially true for FTATip, the most constraining approach in terms of DoFs. Indeed, on average, with this guidance method, the execution time of the participants was reduced by $37 \%$ compared to Ref. This is mostly due to the inherent design of this haptic guide. When the assisted task starts, the guide automatically pulls the user smoothly towards the target and corrects the orientation of the needle, while ensuring that the tip remains close to the normal of the tissue surface. Thus, the user is drawn in the right direction straight away and is able to reach the desired position and orientation quicker than with a lower level of constraint. On the other hand, without assistance, finding a correct initial orientation is complicated, especially during the first few trials of the participant. This could also partly be attributed to the type of visual feedback that was displayed on the screen. It required a lot of coordination to position the needle in two different views simultaneously. One idea for improvement would be to perform the same experiment in a $3 \mathrm{D}$ environment, thanks to a Head-Mounted Display (HMD). The second observation from our user study is the better results obtained when the assistance is applied to the tip of the needle compared to the assistance applied to the effector, thus validating $\mathbf{H}_{\mathbf{B}}$. This observation is particularly interesting for needle pre-positioning and could be further explored for needle insertion.

As for user-experience, the results show that the participants had a preference for assisted targeting tasks rather than unassisted tasks. To illustrate, the unassisted reference gesture (Ref) was marked $2.9 / 7$ on average, whereas the marks received by the five haptic guides were all above 4.3/7. Furthermore, as shown in Table II, $50 \%$ of the panel of participants chose the most constraining guidance method, FTATip, as their favourite. This is also illustrated by the participants' answers in the questionnaire, which bring FTATip forward. On average, this haptic guide was marked 6.2/7. Though, for some participants, FTATip was not comfortable enough. Those $(33.3 \%)$ usually preferred TTip, which was deemed precise, but also, more comfortable to use because it imposes less constraints to the user. Finally, at the end of the user study, two distinct panels appeared, with about half of the participants in each. The first one included those who preferred a higher level of constraint and the second one, those who would rather choose a more flexible guidance. This subjective study highlights that some users are more willing to delegate parts of the intervention to the robot, than others. In the end, FTATip appears as the best in terms of performance and user experience.

\section{CONCLUSION AND FUTURE WORK}

This paper focuses on the design and experimental comparison of five haptic guides dedicated to pre-positioning assistance of a comanipulated needle. Those provide the user with assistance in the form of haptic cues about the current state of the needle and/or its positioning error with respect to its planned trajectory. An experimental validation of the guides was conducted through a user study involving twelve participants. The goal was to compare the five haptic guides and the unassisted reference gesture. The criteria were taskexecution time and user experience. The results demonstrated that the more the user is constrained by the guide, the faster he/she completes the targeting task. Then, it was measured that the assistance applied to the tip of the needle helped the user complete the task faster and provided a higher level of user experience than that applied to the end-effector of the haptic device for pre-positioning the needle before its insertion. Finally, the most constraining guide, FTATip, yielded a $37 \%$ reduction of the execution time, compared to the unassisted reference gesture, and received the highest average mark of $6.2 / 7$ in the subjective questionnaire. Thus, it was highlighted as the best guide in terms of performance and user experience for pre-positioning a needle. Those promising results open possibilities for increasing the level 


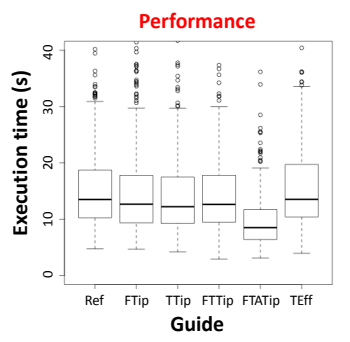

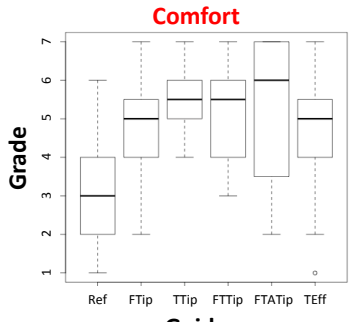

Guide

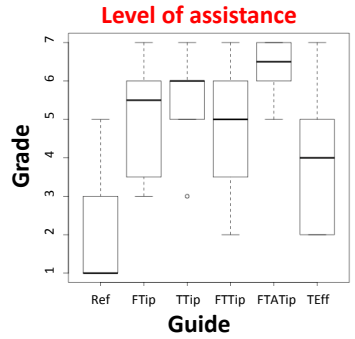

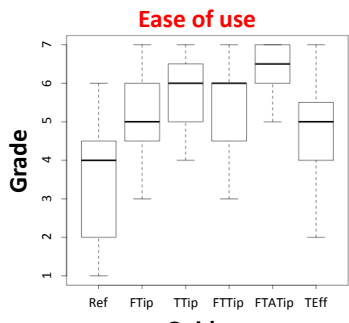

Guide

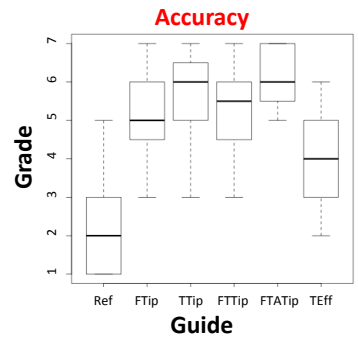

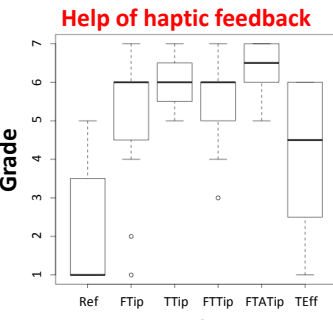

Guide

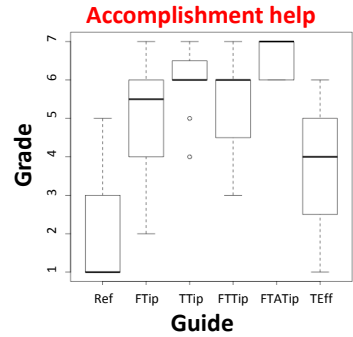

Fig. 3. Box plots showing the results of the user study: (a): Performance (execution time) (b): Answers to the subjective questionnaire, using a 7-point Likert scale. The following criteria are considered: Comfort, Ease of use, Help of haptic feedback, Level of assistance, Accuracy and Accomplishment help. Each box plot is delimited by the quartile (25\% quantile and $75 \%$ quantile) of the distribution of the effect over the individuals. The median is also represented for each criterion.

of accuracy and reliability of needle pre-positioning and preorienting. It also paves the way for the future design of efficient haptic guides dedicated to comanipulated needle insertion in soft tissues.

\section{REFERENCES}

[1] N. Abolhassani and R.V. Patel. Deflection of a flexible needle during insertion into soft tissue. In Proc. of the 28th IEEE Engineering in Medicine and Biology Society, pages 3858-3861, 2006.

[2] A.M. Okamura, C. Simone, and M.D. O'Leary. Force modeling for needle insertion into soft tissue. IEEE Trans. on Biomedical Engineering, 51(10):1707-1716, October 2004.

[3] D.R. Kaye, D. Stoianovici, and M. Han. Robotic ultrasound and needle guidance for prostate cancer management: review of the contemporary literature. Current Opinion in Urology, 24(1):75-80, January 2014.

[4] L.B. Rosenberg. Virtual Fixtures: Perceptual Overlays Enhance Operator Performance in Telepresence Tasks. PhD thesis, Stanford University, Stanford, CA, USA, 1994. UMI Order No. GAX95-08441.

[5] S.A Bowyer, B.L. Davies, and R.F. Baena. Active constraints/virtual fixtures: A survey. IEEE Trans. on Robotics, 30(1):138-157, 2014.

[6] S.C. Ho, R.D. Hibberd, and B.L. Davies. Robot assisted knee surgery. IEEE Engineering in Medicine and Biology Magazine, 14(3):292-300, 1995.

[7] B.L. Davies, K.L. Fan, R.D. Hibberd, M. Jakopec, and S.J. Harris. Acrobot-using robots and surgeons synergistically in knee surgery. IEEE Int. Conference on Advanced Robotics, pages 173-178, 1997.

[8] L. Xiong, C.B. Chng, C.K. Chui, P. Yu, and Y. Li. Shared control of a medical robot with haptic guidance. The Int. Journal of Computer Assisted Radiology and Surgery, 12(1):137-147, January 2017.

[9] L. Meli, C. Pacchierotti, and D. Prattichizzo. Experimental evaluation of magnified haptic feedback for robot-assisted needle insertion and palpation. The Int. Journal of Medical Robotics and Computer Assisted Surgery, 2017.

[10] M.-A. Vitrani, C. Poquet, and G. Morel. Applying virtual fixtures to the distal end of a minimally invasive surgery instrument. IEEE Trans. on Robotics, pages 1-10, 2017.

[11] R. Chalard, D. Reversat, G. Morel, P. Mozer, and M.-A. Vitrani. Precisely positioning the tip of an instrument inserted through an orifice with a free wrist robot: application to prostate biopsies. The Int. Journal of Computer Assisted Radiology and Surgery, 13:611-618, 2018.

[12] A. Gijbels, J. Smits, L. Schoevaerdts, K. Willekens, E.B. Vander Poorten, P. Stalmans, and D. Reynaerts. In human robot assisted retinal vein cannulation, a world first. Annals of Biomedical Engineering, 46(10):1676-1685, October 2018.
[13] M. Wartenberg, J. Schornak, K. Gandomi, P. Carvalho, C. Nycz, N. Patel, I. Iordachita, C. Tempany, N. Hata, J. Tokuda, and G.S. Fischer. Closed-loop active compensation for needle deflection and target shift during cooperatively controlled robotic needle insertion. Annals of Biomedical Engineering, 46(10):1582-1594, October 2018.

[14] S. Xu, V. Krishnasamy, E. Levy, M. Li, Z.T.H. Tse, and B.J. Wood. Smartphone-guided needle angle selection during ct-guided procedures. American Journal of Roentgenology, 210(1):207-213, January 2018.

[15] P. Durand, A. Moreau-Gaudry, A.-S. Silvent, J. Frandon, E. Chipon, M. Médici, and I. Bricault. Computer assisted electromagnetic navigation improves accuracy in computed tomography guided interventions: A prospective randomized clinical trial. PLOS ONE, 12(3), March 2017.

[16] R. F. Grasso, E. Faiella, G. Luppi, E. Schena, F. Giurazza, R. Del Vescovo, F. D'Agostino, R. L. Cazzato, and B. Beomonte Zobel. Percutaneous lung biopsy: comparison between an augmented reality ct navigation system and standard ct-guided technique. The Int. Journal of Computer Assisted Radiology and Surgery, 8(5):837848, September 2013.

[17] N. Hungr, M. Baumann, J.-A. Long, and J. Troccaz. A 3-d ultrasound robotic prostate brachytherapy system with prostate motion tracking. IEEE Trans. on Robotics, 28(6):1382-1397, December 2012.

[18] Renishaw. NeuroMate, 1999.

[19] Perfint Healthcare. Maxio, 2014.

[20] G.-Z. Yang, J. Cambias, K. Cleary, E. Daimler, J. Drake, P.E. Dupont, N. Hata, P. Kazanzides, S. Martel, R.V. Patel, V.J. Santos, and R.H. Taylor. Medical robotics-regulatory, ethical, and legal considerations for increasing levels of autonomy. Science Robotics, 2(4), 2017.

[21] M.-A. Vitrani, M. Baumann, D. Reversat, G. Morel, A. MoreauGaudry, and P. Mozer. Prostate biopsies assisted by comanipulated probe-holder: first in man. The Int. Journal of Computer Assisted Radiology and Surgery, pages 1-9, 2016.

[22] Haption. Virtuose ${ }^{T M} 6 D, 2001$.

[23] Z. Yaniv. Which pivot calibration? In Robert J. Webster and Ziv R. Yaniv, editors, SPIE Medical Imaging, March 2015.

[24] W. Schroeder, K. Martin, and B. Lorensen. The visualization toolkit. In VTK Textbook. Kitware, 2006.

[25] B.K.P. Horn. Closed-form solution of absolute orientation using unit quaternions. Journal of the Optical Society of America A, 4(4):629, April 1987. 